03

\title{
Барические изменения параметров оптической индикатрисы кристаллов литий натрий сульфата
}

\author{
(C) В.И. Стадник ${ }^{1}$, П.А. Щепанский ${ }^{1,2}$, Р.С. Брезвин ${ }^{1}$, \\ М.Я. Рудиш ${ }^{1,2}$, Р.Б. Матвиив ${ }^{1}$ \\ ${ }^{1}$ Львовский национальный университет им. Ивана Франко, \\ 79005 Львов, Украина \\ 2 Ченстоховский университет им. Я. Длугоша, \\ 75-453 Ченстохова, Польша \\ e-mail: vasylstadnyk@ukr.net
}

Поступила в редакцию 06.02.2019 г.

В окончательной редакции 17.08.2019 г.

Принята к публикации 20.08.2019 г.

Изучены спектральные, температурные и барические зависимости двулучепреломления $\Delta n_{i}$ кристаллов $\mathrm{LiNaSO}_{4}$ и установлено, что дисперсия $\Delta n(\lambda)$ аномальная. Показано, что одноосное сжатие приводит к разным по величине и знаку изменениям $\Delta n_{i}$. Дисперсионный эффект изменений $\Delta n_{i}$ уменьшается под влиянием напряжения $\sigma \perp z$ и увеличивается в противоположном случае $\sigma \| z$. Оценены барические изменения показателей преломления и установлено, что при действии одноосного сжатия показатели преломления кристалла ЛНС увеличиваются, что может быть связано с сжатием кристалла и изменением природы его межатомной связи.

Ключевые слова: показатели преломления, двулучепреломление, одноосное сжатие, кристаллы литий натрий сульфата, дисперсия, анизотропия.

DOI: $10.21883 /$ OS.2019.12.48688.46-19

\section{Введение}

Кристаллы литий-аммоний сульфата $\mathrm{LiNaSO}_{4}$ (ЛНС) при комнатной температуре ( $\beta$-модификация) имеют [1] тригональную симметрию (пространственная группа $P 31 c)$ и шесть формульных единиц в элементарной ячейке (параметры ячейки $a=7.6310 \AA$ и $c=9.8600 \AA$ ). Элементарная ячейка имеет вид правой призмы, вершины и центры ребер которой ориентированы вдоль оси $c$ и заняты атомами серы. Каждый ион $\mathrm{S}^{+6}$ окружен четырьмя ионами $\mathrm{O}^{-2}$, которые образуют правильные тетраэдры. Среднее расстояние $\mathrm{S}-\mathrm{O}(1.469 \AA)$ для ЛНС согласуется с известными параметрами (1.467-1.474 $\AA$ ) для кристаллов сульфатов [1-3]. Структура $\beta$-ЛНС построена из тетраэдров $\mathrm{SO}_{4}$ и $\mathrm{LiO}_{4}$, соединенных своими вершинами $[1,3]$. Структуру $\beta$-ЛНС также можно представить как „сандвич“ - поочередное соединение плоскостей двух типов. Шары первого типа заняты ионами $\mathrm{Li}^{+}$и $\mathrm{S}^{+6}$, другие шары - ионами $\mathrm{Na}^{+}$и $\mathrm{S}^{+6}$.

Выше $788 \mathrm{~K}$ кристалл ЛНС имеет высокотемпературную ( $\alpha$-модификация) объемо-центрированную кубическую структуру с высоким разупорядочением катионных $\left(\mathrm{Li}^{+}, \mathrm{Na}^{+}\right)$и кислородных подрешеток [4,5]. Было показано, что ионы $\mathrm{Li}^{+}, \mathrm{Na}^{+}$и $\mathrm{O}^{-2}$ не имеют фиксированных координат в структуре $\alpha$-ЛНС, поэтому высокотемпературную фазу ЛНС иногда рассматривают как квазижидкое состояние. В $\alpha$-модификации $(788-888 \mathrm{~K})$ кристаллы ЛНС обладают очень высокой ионной проводимостью (ниже точки плавления $888 \mathrm{~K}$ проводимость составляет $\left.1.35 \Omega^{-1} \cdot \mathrm{cm}^{-1}\right)[6,7]$.

В кристалле ЛНС исследованы электрические [8-10], термические $[11,12]$, люминесцентные $[13,14]$ свойства, ядерный магнитный резонанс, инфракрасные и рамановские спектры [15-18]. Установлено, что кристаллы ЛНС - это оптически двупреломляющий одноосный кристалл [19]. Показатели преломления при нормальном давлении и комнатной температуре равны $n_{\gamma}=1.497$ и $n_{\alpha}=1.488(\lambda=589.29 \mathrm{~nm})[3]$.

В последнее время возобновляется интерес к этим кристаллам в связи с возможностью их широкого использования. Кристаллы ЛНС нечувствительны к влажности, имеют высокую ионную проводимость и сравнительно недорогие и их можно легко приготовить из водного раствора щелочных сульфатов. Эти свойства дают возможность использовать эти кристаллы в твердотельных батареях, топливных элементах и резервных батареях. Наиболее перспективным применением ЛНС является твердый электролит у резервных батарей для военной техники (например, ракет).

Ранее исследовались спектральные зависимости показателей преломления $n_{i}(\lambda)$ [20]. Установлено, что дисперсия $n_{i}(\lambda)$ нормальная $\left(d n_{i} / d \lambda<0\right)$, а соотношения $n_{x}=n_{y} \neq n_{z}$ и $n_{z}>n_{x, y}$ справедливы для всей исследуемой области спектра, что подтверждает тот факт, что ЛНС является оптически одноосным и положительным [8]. Однако в литературе отсутствуют сведения о влиянии механических напряжений на рефрактивные 
свойства данных кристаллов. Поэтому целью настоящей работы является экспериментальное исследование барических зависимостей показателей преломления и двулучепреломления кристалла $\mathrm{LiNaSO}_{4}$ в широком спектральном диапазоне, а также анализ их пьезооптических свойств.

\section{Методика эксперимента}

Исследуемый кристалл $\mathrm{LiNaSO}_{4}$ был синтезирован из водного стехиометрического раствора солей $\mathrm{Li}_{2} \mathrm{SO}_{4} \cdot \mathrm{H}_{2} \mathrm{O}$ и $\mathrm{Na}_{2} \mathrm{SO}_{4}$ в дистиллированной воде путем испарения при комнатной температуре:

$$
\mathrm{Li}_{2} \mathrm{SO}_{4} \cdot \mathrm{H}_{2} \mathrm{O}+\mathrm{Na}_{2} \mathrm{SO}_{4} \rightarrow 2 \mathrm{LiNaSO}_{4}+\mathrm{H}_{2} \mathrm{O}
$$

Синтезированные кристаллы были хорошего оптического качества, имели хорошо развитую огранку. Ориентация кристаллов проводилась с помощью поляризационного микроскопа и за видом коноскопических картин.

В спектральном диапазоне 250-800 nm дисперсия двулучепреломления изучалась по изменениям интерференционной картины поляризованных лучей, что регистрировалось с помощью спектрального комплекса КСВУ-23. Исследуемый образец устанавливался в криостат в диагональное положение между скрещенных николей. При прохождении через такую систему пучка белого света в фокальной плоскости спектрографа возникала интерференционная картина. Условием появления интерференционных экстремумов в спектре является равенство:

$$
d\left(n_{i}-n_{j}\right)=k \lambda,
$$

где $k$ - порядок интерференционного максимума, $d-$ толщина образца по ходу лучей, $n_{i}$ и $n_{j}$ - показатели преломления в кристаллофизических направлениях $i$ и $j$. Порядок интерференционной картины определялся по известным значениям показателя преломления.

В исследуемом спектральном диапазоне на КСВУ-23 записывалась спектральная зависимость интенсивности пропускания скрещенных поляризаторов и образца между ними в диагональном положении согласно известному соотношению:

$$
I=I_{0} \sin ^{2} \frac{\pi}{\lambda} \Delta n_{i} d_{i},
$$

где $I_{0}$ - интенсивность света, который падает на образец; $\Delta n_{i}, d_{i}-$ величина двулучепреломления и толщина образца в направлении просвечивания. За экстремумами интерференционной картины находим спектральную зависимость разности хода (1).

При одноосном сжатии образца, которое осуществлялось с помощью специального приспособления, наблюдается спектральное смещение интерференционных полос, за которое отвечает изменение двулучепреломления. Зависимость величины $\Delta n_{i}$ от давления при этом будет определяться выражением [21,22]:

$$
\Delta n_{i}\left(\lambda, \sigma_{i}\right)=k \lambda / d(\sigma)
$$

Изменения толщины при действии одноосного напряжения $d(\sigma)$ можно рассчитать, исходя из изменений линейных размеров образца вдоль направления сжатия, полученных на основе закона Гука

$$
\left(\Delta l / l_{0}\right)_{i}=s_{i m} \sigma_{m},
$$

где $\Delta l / l_{0}-$ коэффициенты относительного сжатия, $s_{i m}$ - коэффициенты тензора упругой податливости. Поскольку значения коэффициентов $s_{i m}$ для кристаллов ЛНС находятся в пределах $\sim 10^{-11} \mathrm{~m}^{2} / \mathrm{N}$, то вклад от $d(\sigma) \sim \Delta l / l_{0}$ в барические изменения двулучепреломления пренебрежимо мал.

Измерения проводились до давлений $\sigma_{m} \sim 200$ bar. Погрешность измерения двулучепреломления составляла $\delta \Delta n_{i}= \pm 2 \cdot 10^{-5}$.

\section{Результаты и их обсуждение}

\section{а) температурно-спектральные зависимости двулучепреломления механически свободных кристаллов}

На рис. 1 показана спектральная зависимость двулучепреломления кристаллов ЛНС для разных значений температур. Подобно до изоморфного кристалла $\mathrm{LiKSO}_{4}$ [23] дисперсия $\Delta n(\lambda)$ ЛНС аномальная $(d(\Delta n) / d \lambda>0)$ и незначительно изменяется в видимом диапазоне $\left(d(\Delta n) / d \lambda=1.9 \cdot 10^{-6}\right.$ для $400 \mathrm{~nm}$, $1.2 \cdot 10^{-6}$ для $750 \mathrm{~nm}$ и $1.3 \cdot 10^{-6}$ в среднем). Следует отметить достаточно высокую для кристаллов группы $\mathrm{ABSO}_{4}$ оптическую анизотропию ЛНС: только $\left(\mathrm{NH}_{4}\right)_{2} \mathrm{SO}_{4}$ и $\beta$ - $\mathrm{LiNH}_{4} \mathrm{SO}_{4}$ имеют еще большую анизотропию.

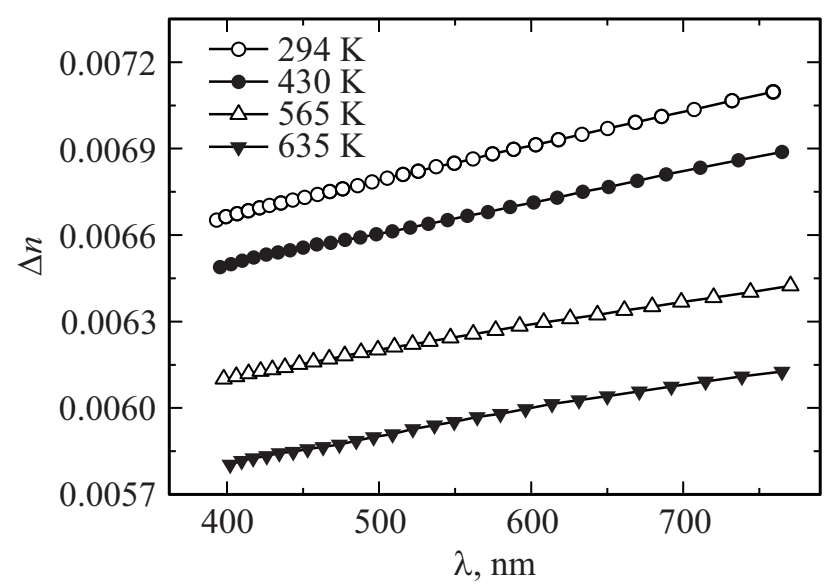

Рис. 1. Спектральная зависимость двулучепреломления $\Delta n(\lambda)$ кристаллов ЛНС для разных значений температур. 
Таблица 1. Температурно-спектральные параметры двулучепреломления $\Delta n(\lambda, T)$ кристаллов ЛНС

\begin{tabular}{|c|c|c|c|c|}
\hline \multirow{2}{*}{ Температура, K } & \multirow{2}{*}{$\begin{array}{c}d(\Delta n) / d T, 10^{-5} \mathrm{~K}^{-1} \\
(\text { при } \lambda=500 \mathrm{~nm})\end{array}$} & \multicolumn{3}{|c|}{$d(\Delta n) / d \lambda, 10^{-6} \mathrm{~nm}^{-1}$} \\
\hline & & $\lambda=400 \mathrm{~nm}$ & $\lambda=550 \mathrm{~nm}$ & $\lambda=750 \mathrm{~nm}$ \\
\hline $\begin{array}{l}294 \\
430 \\
565 \\
635\end{array}$ & $\begin{array}{l}-0.7 \\
-1.9 \\
-3.9 \\
-4.8\end{array}$ & $\begin{array}{l}1.70 \\
1.53 \\
1.13 \\
1.7\end{array}$ & $\begin{array}{l}1.12 \\
1.06 \\
0.87 \\
1.01\end{array}$ & $\begin{array}{l}1.14 \\
1.01 \\
0.81 \\
0.66\end{array}$ \\
\hline
\end{tabular}

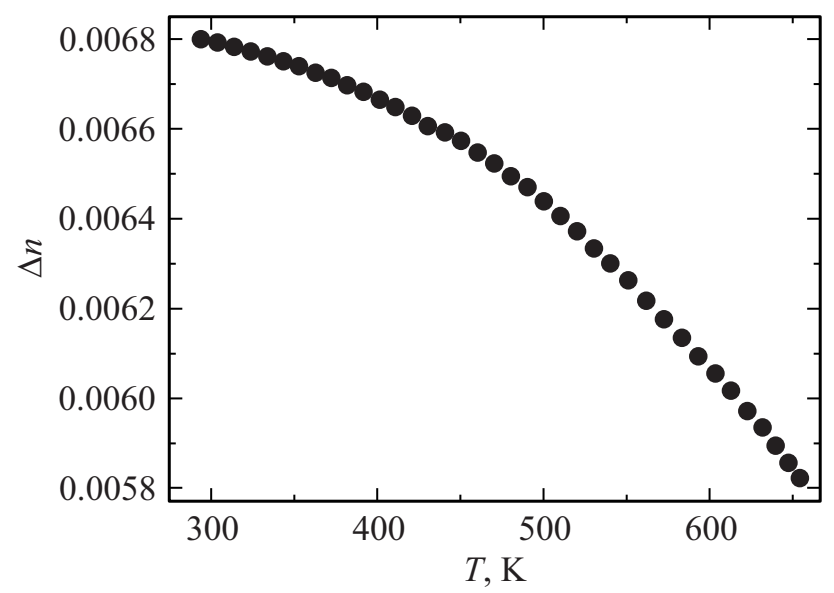

Рис. 2. Температурная зависимость двулучепреломления $\Delta n(T)$ кристаллов ЛНС для длины волны $\lambda=500 \mathrm{~nm}$.

Нами рассчитано среднее значение двулучепреломления $\overline{\Delta n}=\left(\left|\Delta n_{x}\right|+\left|\Delta n_{y}\right|+\left|\Delta n_{z}\right|\right) / 3$, типичное для кристаллов ЛНС, $\mathrm{K}_{1.75}\left(\mathrm{NH}_{4}\right)_{0.25} \mathrm{SO}_{4}$ и $\mathrm{LiKSO}_{4}$ при $500 \mathrm{~nm}[3,23,24]$. Параметр $\overline{\Delta n}$ для ЛНС (0.0043) несколько больше, чем для $\mathrm{K}_{1.75}\left(\mathrm{NH}_{4}\right)_{0.25} \mathrm{SO}_{4}(0.0028)$ и более чем на порядок больше, чем для $\mathrm{LiKSO}_{4}(0.0004)$. Можно предположить, что большая разница в двулучепреломлении структурно очень близких $\mathrm{LiNaSO}_{4}$ и $\mathrm{LiKSO}_{4}$ является следствием различия в структурной анизотропии, а именно существенно различных вторых координационных окружений аниона, а также анизотропии параметров решетки $\Delta a$ $(\Delta a=(|a-b|+|a-c|+|b-c|) / 3)$.

Из рис. 1 также видно, что оптическая анизотропия уменьшается при повышении температуры. Усреднение данных для разных длин волн (табл. 1) свидетельствует о том, что дисперсионные изменения $\Delta n(\lambda, T)$ также уменьшаются при повышении температуры.

На рис. 2 показана температурная зависимость $\Delta n(T)$ кристаллов ЛНС для фиксированной длины волны $(\lambda=500 \mathrm{~nm})$. Видно, что данная зависимость является нелинейной. Ее количественные характеристики также приведены в табл. 1. Следует отметить, что $\Delta n_{i}$ изоморфного кристалла $\mathrm{LiKSO}_{4}$ увеличивается при повышении температуры [15], тогда как поведение $\Delta n_{i}$ для ЛНС противоположно. Можно предположить, что уменьше- ние оптической анизотропии при высоких температурах является общей тенденцией, обусловленной тем, что структура ЛНС постепенно превращается в его кубическую версию, характерную для высокотемпературной $\alpha$-фазы. В связи с этим следует отметить, что изотропная точка, которая наблюдается в $\mathrm{LiKSO}_{4}$, смещается от 240 до $360 \mathrm{~nm}$ в широком температурном интервале (180-420 K [25]). Поэтому можно предположить, что изотропное состояние в ЛНС следует искать при существенно более высоких температурах в коротковолновой области спектра.

\section{б) барические изменения двулучепреломления кристаллов $\mathrm{LiNaSO}_{4}$}

На рис. 3 показаны спектральные зависимости двулучепреломления кристаллов ЛНС при комнатной температуре, одноосно зажатых давлениями параллельно и перпендикулярно оптической оси.

Как видно из рис. 3 , одноосные сжатия параллельно и перпендикулярно до оси $z$ кристаллов ЛНС приводят к практически одинаковым по абсолютной величине изменениям двулучепреломления, но противоположным по знакам. Следует отметить, что увеличение (или уменьшение) оптической анизотропии, которое наблюдается под действием механического сжатия параллельно (или

Таблица 2. Характеристики барических изменений дисперсии двулучепреломления $\Delta n(\lambda)$ для кристаллов ЛНС при температуре $T=294 \mathrm{~K}$

\begin{tabular}{|c|c|c|c|c|c|}
\hline \multirow{2}{*}{$\begin{array}{r}\text { Длина } \\
\text { волны } \\
\lambda, \mathrm{nm}\end{array}$} & \multirow{2}{*}{$\begin{array}{c}\text { Направление } \\
\text { распространения } \\
\text { света }\end{array}$} & \multicolumn{4}{|c|}{$\begin{array}{c}-d(\Delta n) / d \lambda, 10^{-6} \mathrm{~nm}^{-1} \\
\left(\sigma_{i}=200 \text { bar }\right)\end{array}$} \\
\hline & & $\sigma=0$ & $\sigma_{x}$ & $\sigma_{y}$ & $\sigma_{z}$ \\
\hline \multirow{2}{*}{400} & $X$ & \multirow{2}{*}{1.70} & - & 1.56 & \multirow{2}{*}{1.72} \\
\hline & $Y$ & & 1.56 & - & \\
\hline \multirow{2}{*}{550} & $X$ & \multirow{2}{*}{1.12} & - & 1.11 & \multirow{2}{*}{1.27} \\
\hline & $Y$ & & 1.11 & - & \\
\hline \multirow{2}{*}{750} & $X$ & \multirow{2}{*}{1.14} & - & 1.02 & \multirow{2}{*}{1.16} \\
\hline & $Y$ & & 1.02 & - & \\
\hline
\end{tabular}


перпендикулярно) оптической оси, также является характерным для одноосных кристаллов $\mathrm{LiKSO}_{4}$ [25]. Дисперсионный эффект у соответствующей кривой $\Delta n(\lambda)$ для ЛНС ослабляется под действием напряжения $\sigma \perp z$ и усиливается под действием $\sigma \| z$ (табл. 2). Из рис. 4 видно, что барические зависимости двулучепреломления $\Delta n(\sigma)$ практически линейны. Сравнение с кристаллами $\left(\mathrm{NH}_{4}\right)_{2} \mathrm{SO}_{4}$ и $\mathrm{RbKSO}_{4}$ [26] показывает, что двулучепреломление ЛНС практически на порядок менее чувствительно к механическим напряжениям.

Используя индуцированные одноосными напряжениями изменения двулучепреломления, известную формулу

$$
\pi_{i m}^{0}=\frac{2 \delta \Delta n_{i}}{\sigma_{m}}-2 \Delta n_{i} s_{i m}
$$

и известную методику $[27,28]$, нами рассчитаны дисперсионные зависимости пьезооптических коэффициентов исследуемых кристаллов (рис. 5). Здесь $\delta n_{i}-$ индуцированное изменение двулучепреломления для направления распространения света вдоль кристаллофизической оси $i$ и направления сжатия кристалла вдоль оси $m$. Второе слагаемое учитывает изменение размеров образцов вдоль оси $i, s_{i m}-$ коэффициент упругой податливости.

Видно, что при комнатной температуре дисперсионная зависимость коэффициентов $\pi_{i m}^{0}$ слаба, при этом дисперсия $\pi_{13}^{0}$ является нормальной, а $\pi_{12}^{0}-$ аномальной. Дисперсионное изменение пьезокоэффициента $\pi_{13}^{0}$ составляет $12.9 \cdot 10^{-15}$ для $400 \mathrm{~nm}$ и $2.2 \cdot 10^{-15}$ для 700 , а $\pi_{12}^{0}-7.1 \cdot 10^{-15}$ и $-4.8 \cdot 10^{-15}$ соответственно для 400 и $700 \mathrm{~nm}$. Разные знаки и спектральные изменения $\pi_{i m}^{0}$ указывают на то, что влияние одноосного механического напряжения вдоль кристаллофизических осей $x=y$ и $z$ приводит к разному характеру изменений индуцированного двулучепреломления кристалла. Дисперсионные изменения пьезокоэффициентов $\pi_{12}^{0}$ и $\pi_{13}^{0}$ кристаллов ЛНС и $\alpha-\mathrm{LiNH}_{4} \mathrm{SO}_{4}$ схожи по своему характеру, однако абсолютные значения констант сильно различаются: среднее $\pi_{12}^{0}=5.6 \mathrm{~B}$ для $\alpha$ - $\mathrm{LiNH}_{4} \mathrm{SO}_{4}$, тогда как для ЛНС $\pi_{12}^{0}=-2.1 \mathrm{~B}, \pi_{13}^{0}=-5.8 \mathrm{~B}$ для $\alpha-\mathrm{LiNH}_{4} \mathrm{SO}_{4}$ и $2.2 \mathrm{~B}$ для ЛНС.

Используя известные соотношения между пьезооптическими коэффициентами и показателями преломления:

$$
\begin{gathered}
\pi_{i m}^{0}(\lambda)=\pi_{i m}(\lambda) n_{m 0}^{3}(\lambda)-\pi_{j m}(\lambda) n_{j 0}^{3}(\lambda), \\
\delta n_{i}(\lambda)=\frac{1}{2} \pi_{m}(\lambda) \sigma_{m} n_{i 0}^{3}(\lambda),
\end{gathered}
$$

нами были оценены барические изменения показателей преломления $\delta n_{i}$ (здесь $n_{i 0}-$ показатель преломления механически свободного кристалла ЛНС); $\pi_{i m}$ - абсолютные пьезооптические коэффициенты, рассчитанные на основе полученных барических зависимостей двулучепреломления. Установлено, что под действием одноосных напряжений величиной до $200 \mathrm{bar}$ показатели преломления увеличиваются в среднем на

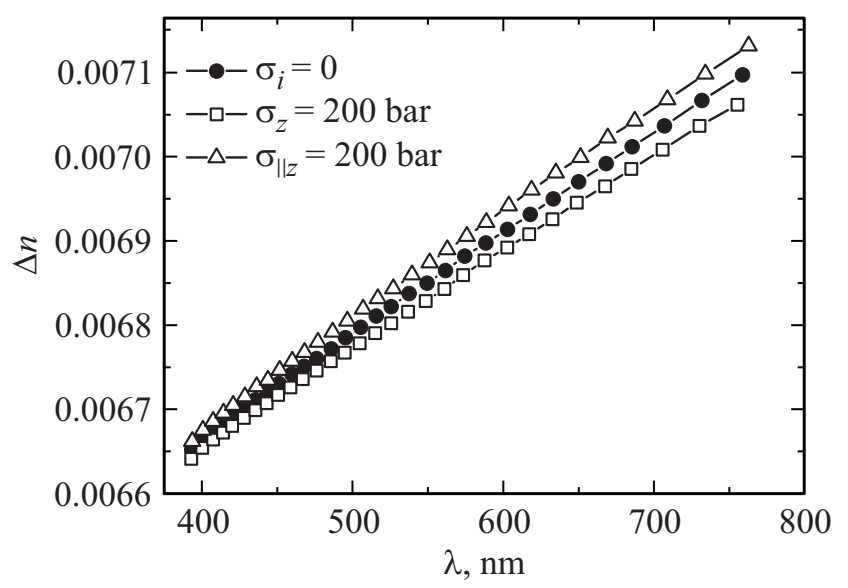

Рис. 3. Дисперсия двулучепреломления $\Delta n(\lambda)$ кристаллов ЛНС для разных направлений одноосного сжатия при комнатной температуре.

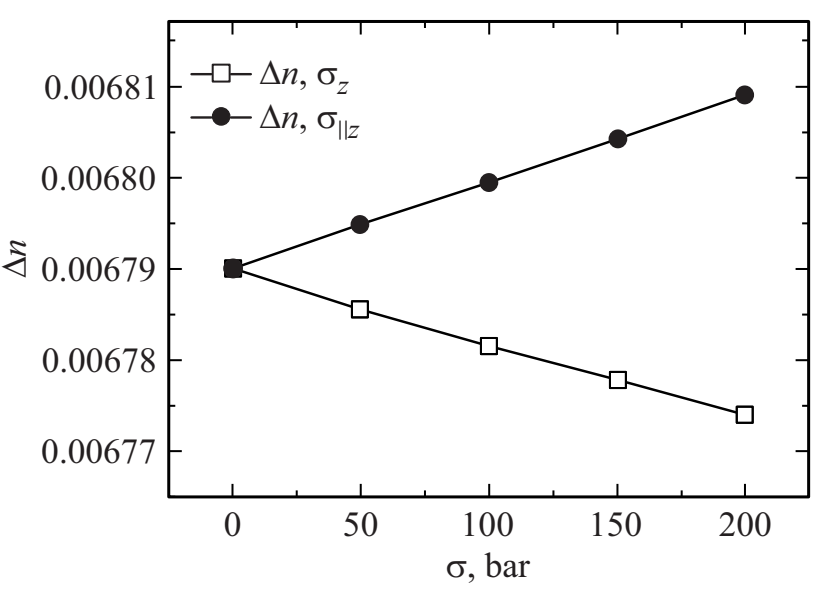

Рис. 4. Барическая зависимость двулучепреломления $\Delta n(\sigma)$ для разных направлений одноосного сжатия для $\lambda=500 \mathrm{~nm}$ и комнатной температуры $(T=294 \mathrm{~K})$.

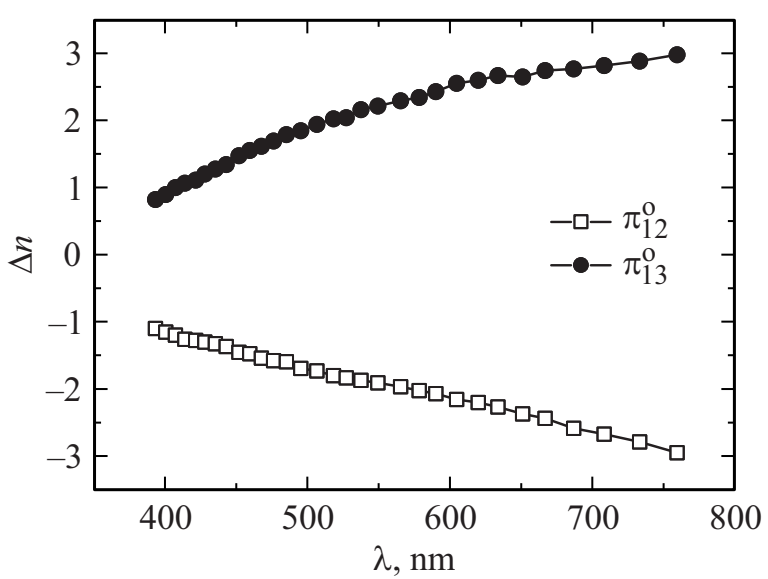

Рис. 5. Спектральная зависимость комбинированных пьезооптических коэффициентов кристаллов ЛНС при комнатной температуре. 
$(1.9-2.1) \cdot 10^{-4}$ или $\partial n_{i} / \partial \sigma \approx 2 \cdot 10^{-6} \operatorname{bar}^{-1}$ для исследуемого спектрального диапазона.

Исходя из дисперсионной формулы Лорентц-Лоренца, которая описывает рефракцию $R$ и электронную поляризуемость $\alpha$ кристаллов [29]:

$$
R=4 \pi / 3 N_{A} \alpha=\frac{\mu}{\rho} \frac{n^{2}-1}{n^{2}+2},
$$

для случая одноосного сжатия можно записать

$$
\begin{aligned}
\frac{d R}{d \sigma}= & -\left(\frac{\mu}{\rho^{2}} \frac{n^{2}-1}{n^{2}+2} \frac{d \rho}{d \sigma}\right)_{n} \\
& +\left(\frac{\mu}{\rho} \frac{6 n}{\left(n^{2}+2\right)^{2}} \frac{d n}{d \sigma}\right)_{N} .
\end{aligned}
$$

Здесь $N$ - число частиц в единице объема, $N_{A}-$ число Авогадро, $\mu$ - молярная масса, $\rho-$ плотность кристалла. Первое слагаемое указывает на изменение числа дисперсионных центров в объеме (через изменение самого объема при сжатии), а второе - характеризирует изменение дисперсионной частоты, т.е. самой поляризуемости через изменение характера химической связи. Используя следующие известные численные значения для кристаллов ЛНС: $n_{i}=1.495, \mu=125.9 \mathrm{~g} / \mathrm{mol}$, $\rho \sim 2.53 \mathrm{~g} / \mathrm{cm}^{3}, \quad \frac{\partial \rho}{\partial \sigma} \cong \rho s_{i m}(1-2 v), \quad$ где $v-$ фактор Пуассона, $v=0.25-0.35$ и $\frac{\partial n}{\partial \sigma} \cong 2 \cdot 10^{-6} \mathrm{bar}^{-1}$, получим для первого слагаемого $5.1 \cdot 10^{-5} \mathrm{bar}^{-1}$, для второго $10.7 \cdot 10^{-5} \mathrm{bar}^{-1}$, т. е. геометрический фактор составляет $\sim 32 \%$ общего барического приращения $R$. Таким образом, при одноосном сжатии имеет место конкуренция двух факторов - сжатие кристалла и вследствие этого увеличение $n_{i}$ и изменение природы межатомной связи.

\section{Выводы}

Изучены спектральные и температурные зависимости двулучепреломления $\Delta n_{i}$ кристаллов $\mathrm{LiNaSO}_{4}$ и установлено, что дисперсия $\Delta n(\lambda)$ ЛНС аномальная $(d(\Delta n) / d \lambda>0)$.

Установлено, что катионное замещение $\mathrm{K} \rightarrow \mathrm{Na}$ $\left(\mathrm{LiKSO}_{4} \rightarrow \mathrm{LiNaSO}_{4}\right)$ в системе кристаллов $\mathrm{ABSO}_{4}$ значительно (более чем на порядок) увеличивает $\Delta n_{i}$ по сравнению с замещениями $\mathrm{K} \rightarrow \mathrm{Rb}$ и $\mathrm{K} \rightarrow \mathrm{NH}_{4}$.

Показано, что высокая оптическая анизотропия кристаллов ЛНС может быть связана с асимметрией, обнаруженной во втором координационном окружении их структуры.

Исследовано поведение двулучепреломления кристаллов $\mathrm{LiNaSO}_{4}$ в условиях одноосных напряжений вдоль главных кристаллофизических направлений и установлено, что одноосное сжатие приводит к разным по величине и знаку изменениям $\Delta n_{i}$. Дисперсионный эффект изменений $\Delta n_{i}$ ослабляется под влиянием напряжения $\sigma \perp z$ и усиливается в противоположном случае $\sigma \| z$.
Оценены барические изменения показателей преломления и установлено, что при действии одноосного сжатия показатели преломления кристалла ЛНС увеличиваются, что, возможно, связано с сжатием кристалла и изменением природы его межатомной связи.

\section{Конфликт интересов}

Авторы заявляют, что у них нет конфликта интересов.

\section{Список литературы}

[1] Morosin B., Smith D.L. // Acta Crystallographica. 1967. V. 22. P. 906.

[2] Freiheit H.C., Kroll H., Putnis A. // Z. Kristallogr. 1998. V. 213. P. 575.

[3] Shchepanskyi P.A., Kushnir O.S., Stadnyk V.Yo., Brezvin R.S., Fedorchuk A.O. // Ukr. J. Phys. Optics. 2018. V. 19. N 3. P. 141.

[4] Forland T., Krogh-Moe J. // Acta Chem. Scand. 1958. V. 11. P. 224.

[5] Polishcuk A.F., Shurzhal T.M. // Electrokhimiya. 1979. V. 9. P. 838.

[6] Karlsson L., McGreevy R.L. // Solid State Ionics. 1995. V. 76. P. 301.

[7] Chen R.H. Tseng Chaw-Ming, Shern C.S., Fukami T. // Solid State Ionics. 2010. V. 181. P. 877.

[8] Singh K., Deshpande V.K. // Solid State Ionics. 1984. V. 13. P. 157.

[9] Gundusharma U.M., MacLean C., Secco E.A. // Solid State Commun. 1986. V. 57. P. 479.

[10] Karppinen M. // Acta Cryst. 2015. B. 71. P. 334.

[11] Pina C.M., Woensdregt C.F. // J. Cryst. 2001. V. 233. P. 355.

[12] Schroeder K., Kvist A., Ljungmark H. // Zeit. Naturforsch. 1972. A. 27. P. 1252.

[13] Sahare P.D., Moharil T.S.V. // Radiation Effects and Defects in Solids. 1990. V. 114. P. 167.

[14] Gupta K.K., Kadam R.M., Dhoble N.S., Lochab, Dhobl S. // Phys. Chem. Chem. Phys. 2018. V. 20. P. 1540.

[15] Junke K-D., Mali M., Roos J., Brinkmann D. // Solid State Ionics. 1988. V. 28-30. P. 1329.

[16] Shakhovoy R.A., Rakhmatullin A., Deschamps M., SarouKanian V., Bessada C. // J. Phys.: Condens. Matter. 2016. V. 28. P. 176003.

[17] Zhang M.I, Putnisb A, Saljea E.K.H. // Solid State Ionics. 2006. V. 177. P. 37.

[18] Teeters D., Frech R.J. // Chem. Phys. 1982. V. 76. P. 799.

[29] Abdulwahab A.M. // Phys. Chem. Solids. 2016. V. 99. P. 11.

[20] Shchepanskyi P.A., Stadnyk V.Yo., Rudysh M.Ya., Brezvin R.S., Andrievskii B.V. // Opt. Spectrosc. 2018. V. 125. N 3. P. 353.

[21] Stadnyk V.Yo., Gaba V.M., Andrievskii B.V., Kohut Z.O. // Phys. Sol. States. 2011. V. 53. N 1. P. 131.

[22] Stadnyk V.Y., Romanjuk M.O. // Phys. Status Solidi (a). 1996. V. 158. P. 289.

[23] Stadnyk V.Yo., Kushnir O.S., Brezvin R.S., Gaba V.M. // Opt. Spectrosc. 2009. V. 106. N 4. P. 614.

[24] Романюк Н.А., Габа В.М., Стадник В.И. // Кристаллография. 1992. Т. 37. В. 4. С. 1039. 
[25] Romanjuk M.O., Stadnyk V.Y. // Ferroelectrics. 1997. V. 192. N 1-4. P. 235.

[26] Stadnyk V.Y., Romanyuk M.O., Tuzyak M.R., Kurlyak V.Yu. // Ukr. J. Phys. 2009. V. 54. N 6. P. 587.

[27] Най Дж. Физические свойства кристаллов. М.: Мир, 1967. $386 \mathrm{c}$.

[28] Нарасимхамурти T. Фотоупругие и электрооптические свойства кристаллов. М.: Мир, 1984. 587 с. 